\title{
Stage I Esophageal Adenocarcinoma AJCC v7
}

National Cancer Institute

\section{Source}

National Cancer Institute. Stage I Esophageal Adenocarcinoma A/CC v7. NCI Thesaurus. Code C8630.

Stage I includes: IA (T1, N0, M0, G1-2, X); IB (T1, N0, M0, G3); (T2, N0, M0, G1-2, GX). T1: Tumor invades lamina propria, muscularis mucosae, or submucosa. T2: Tumor invades muscularis propria. N0: No regional lymph node metastasis. M0: No distant metastasis. G1: Well differentiated. G2: Moderately differentiated. G3: Poorly differentiated. GX: Grade cannot be assessed-stage grouping as G1. (AJCC 7th ed.) 